\title{
Prone Positioning in Acute Respiratory Distress Syndrome
}

\author{
Luciano Gattinoni, MD, FRCP ${ }^{1}$ Mattia Busana, MD ${ }^{1}$ \\ Michael Quintel, MD ${ }^{1}$ \\ 1 Department of Anesthesiology, Emergency and Intensive Care \\ Medicine, University of Göttingen, Göttingen, Germany \\ Semin Respir Crit Care Med 2019;40:94-100.
}

Lorenzo Giosa, $\mathrm{MD}^{1}$

Matteo Maria Macrì, MD¹

\begin{abstract}
Address for correspondence Luciano Gattinoni, MD, FRCP, Department of Anesthesiology, Emergency and Intensive Care Medicine, University of Göttingen, Robert-Koch-Straße 40, 37075 Göttingen, Germany (e-mail: gattinoniluciano@gmail.com).
\end{abstract}

\author{
Abstract \\ Keywords \\ - Acute Respiratory \\ Distress Syndrome \\ - ARDS \\ - prone position \\ - trial design \\ - oxygenation \\ - shape mismatch \\ - chest wall elastance
}

Prone positioning is nowadays considered as one of the most effective strategies for patients with severe acute respiratory distress syndrome (ARDS). The evolution of the pathophysiological understanding surrounding the prone position closely follows the history of ARDS. At the beginning, the focus of the prone position was the improvement in oxygenation attributed to a perfusion redistribution. However, the mechanisms behind the prone position are more complex. Indeed, the positive effects on oxygenation and $\mathrm{CO}_{2}$ clearance of the prone position are to be ascribed to a more homogeneous inflationventilation, to the lung/thoracic shape mismatch, and to the change of chest wall elastance. In the past 20 years, five major trials have tried, starting from different theories, hypotheses, and designs, to demonstrate the effectiveness of the prone position, which finally found its definitive place among the different ARDS supportive therapies.

\section{Introduction}

Among the various therapeutic options in respiratory intensive care, the prone position is the best example of progressive integration of experimental and clinical observations, pathophysiological understanding, and randomized clinical testing. Indeed, the history of the prone position represents the ideal paradigm that should be followed when a new intervention is proposed: the idea has been thought and described, experimentally applied, discussed, refined, and finally tested in progressive clinical trials until finding its definitive place in the therapeutic armamentarium. We now know how, why, and in which patients' proning works.

The first report describing the application of prone positioning in acute respiratory distress syndrome (ARDS) was published in 1976 by Margaret Piehl, who, while testing a special bed (the CircOlectric bed) that allowed a wide range of positional changes up to 180 degrees, rotated the bed until reaching a full pronation. ${ }^{1}$ In five ARDS patients, the $\mathrm{PaO}_{2}$ increased approximately $30 \mathrm{~mm} \mathrm{Hg}$ on average, and this was attributed to a better distribution of the perfusion. The fact that this extreme positional change seemed to be strictly linked to a special device may explain why this report did not immediately impact the intensive care community. In 1977, however, Douglas et al reported the effects of prone positioning in six patients with ARDS in whom an average increase in $\mathrm{PaO}_{2}$ of $69 \mathrm{~mm} \mathrm{Hg}$ was observed after the maneuver. The authors also reported that the improvement in oxygenation was, in part, maintained after turning the patient in the supine position. No changes in ventilation or $\mathrm{PaCO}_{2}$ were observed, and the $\mathrm{PaO}_{2}$ increase was ascribed to a perfusion redistribution. ${ }^{2}$ Then in 1986, Maunder et $\mathrm{al}^{3}$ and our group ${ }^{4}$ reported the first computed tomography (CT) scan images of ARDS, showing a consistent density increase in the dependent lung regions. Both reports carried the striking information that in contrast to the common belief of the time, the radiological densities of lung parenchyma in ARDS were not, as the chest X-ray would suggest, homogeneously distributed throughout the lung parenchyma, but they were mainly present in the dependent lung regions. This observation led to the concept of the "baby lung," 5 and since then, we started turning the patients to the prone position, aiming for a better perfusion of the baby lung that was thought as an anatomical entity located in the nondependent part of the thoracic cage. ${ }^{6}$ Indeed, consistent with the previous reports from Piehl and Douglas and with the perfusion distribution theory, the $\mathrm{PaO}_{2}$ substantially increased in most of the patients when the prone position was applied. However, when we took the first CT scans in the prone position, to our surprise we found that the densities redistributed from the dorsal to the ventral position, ruling out the hypothesis of the baby lung as a
Issue Theme Acute Respiratory Distress Syndrome: Evolving Concepts; Guest Editors: Greg S. Martin, MD, MSc, and Jordan A. Kempker, MD, MSc
Copyright (c) 2019 by Thieme Medical Publishers, Inc., 333 Seventh Avenue, New York, NY 10001, USA. Tel: +1(212) 584-4662.
DOI https://doi.org/ 10.1055/s-0039-1685180. ISSN 1069-3424. 


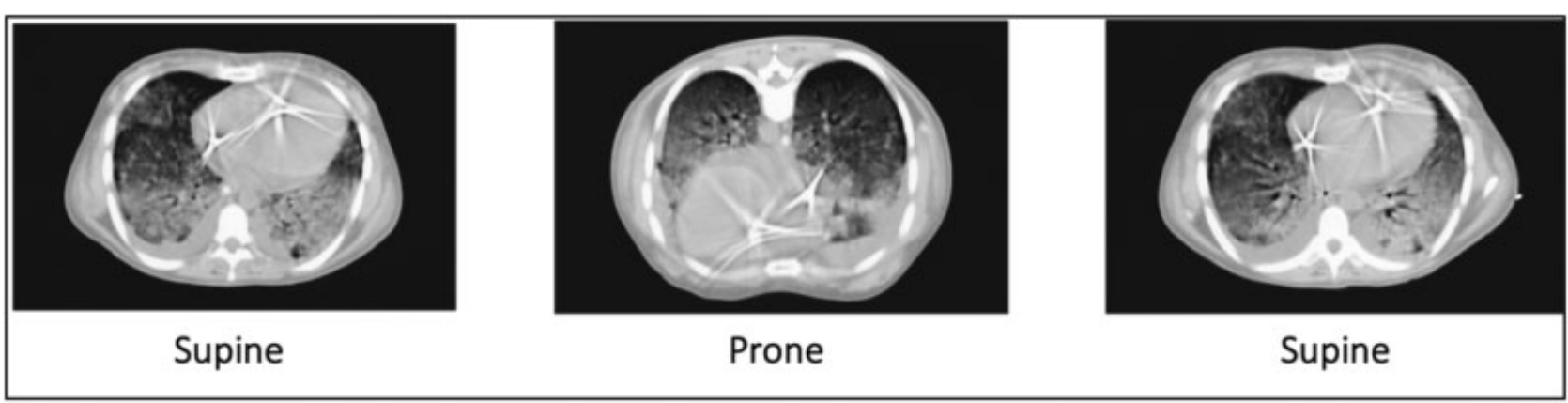

Fig. 1 Computed tomography (CT) scans showing the shift of densities from the dorsal to the ventral regions of the lung during the prone position, and the complete reversal when the patient is turned to the supine position. This led to the idea of the "sponge model."

fixed anatomical compartment and of the blood flow diversion as a primary cause of oxygenation improvement ( $\mathbf{F i g}$. 1). In addition, in the same period, Wiener et $\mathrm{al}^{7}$ showed that the perfusion in the prone position was uniformly distributed throughout the lung, and this observation was confirmed in experimental settings 15 years later. ${ }^{8}$

Therefore, the density redistribution we observed in the prone position pushed us to review the theory behind the oxygenation improvement during prone positioning and to suggest a different explanatory model. This model, which was called "sponge model" by Bone, ${ }^{9}$ gives an explanation for both the density redistribution in the prone position and the maintenance of recruitment with positive end-expiratory pressure (PEEP). ${ }^{10}$ Briefly, in ARDS, the lung weight increases because of the widespread edema, and assuming that the hydrostatic pressures are homogeneously distributed like in a fluid, the increase in lung weight is associated with a progressive increase in the superimposed pressure over the dependent regions of the lung. ${ }^{11}$ This pressure causes the lung collapse through a "squeeze-out" effect of the gas content in the most dependent lung regions, which then results in complete atelectasis. Prone positioning reverses the superimposed pressure so that the dorsal regions "open up" while the ventral ones tend to collapse. The sponge model and the superimposed pressure theory also account for the effect of PEEP: the recruitment is maintained and the lung is kept open when the PEEP provides an intra-alveolar pressure that overcomes the superimposed compressive forces. ${ }^{10}$

\section{Mechanisms of Prone Position and Gas Exchange}

\section{Oxygenation}

Oxygenation may improve through:

- More homogeneous inflation/ventilation distribution.

- Lung mass and shape effects.

- Modification of chest wall elastance.

\section{More Homogeneous Inflation/Ventilation Distribution}

As shown in - Fig. 2, the prone position makes the gas-tissue ratio more homogeneous when compared to the supine position, and this happens both in normal subjects and in patients with ARDS: a more homogeneous distribution of density implies that the transpulmonary distending pres- sures also are more homogeneously distributed in the prone position than in the supine position. Indeed, in the supine position, the nondependent pulmonary units are more distended than the dependent ones, and these differences are dampened when the prone position is applied. The mechanisms through which this happens are likely related to the need of the lung to adapt its shape to the thoracic cage. ${ }^{12}$ As an example, the more "triangular" the nondependent lung when compared to a more "spherical" shape of the thoracic cage, the greater the stretching to line up these two structures. This roughly describes the mechanism called "shape mismatch." The consequence is a greater expansion of the ventral regions compared to the dorsal ones. A further mechanism is linked to the gravitational forces (lung weight), which act by progressively compressing the pulmonary units along the gravitational axis (nondependent $\rightarrow$ dependent). Therefore, in the supine position, both the shape mismatch and the gravitational force act in the same direction (expansion of the nondependent pulmonary units and compression of the dependent ones). These two phenomena lead to lung inhomogeneity both in normal and in ARDS lungs. In the prone position, the shape mismatch and the gravitational forces act in opposing directions: while the shape mismatch tends to expand the ventral regions, the prone position tends to compress them. ${ }^{13}$ The final result is that the expansion of the dependent and nondependent pulmonary units is far more similar in prone than in the supine position. The consequences of this increased homogeneity are evident:

- Ventilation and perfusion are more homogeneously distributed leading to an improvement of oxygenation.

- More importantly, any eventual damage inflicted by the energy load of mechanical ventilation will be more evenly distributed to the lung parenchyma in the prone than in the supine position.

\section{Lung Mass and Shape}

It is usually claimed that the improvement of oxygenation that follows the prone position is because of lung recruitment. This statement, however, is not true. Actually, the lung opening observed in the dorsal regions in the prone position is always associated with a partial closing of the ventral regions. Indeed, when we published the first data on 10 ARDS patients in the prone position, we found that the average densities at the CT scan did not change with the position. 


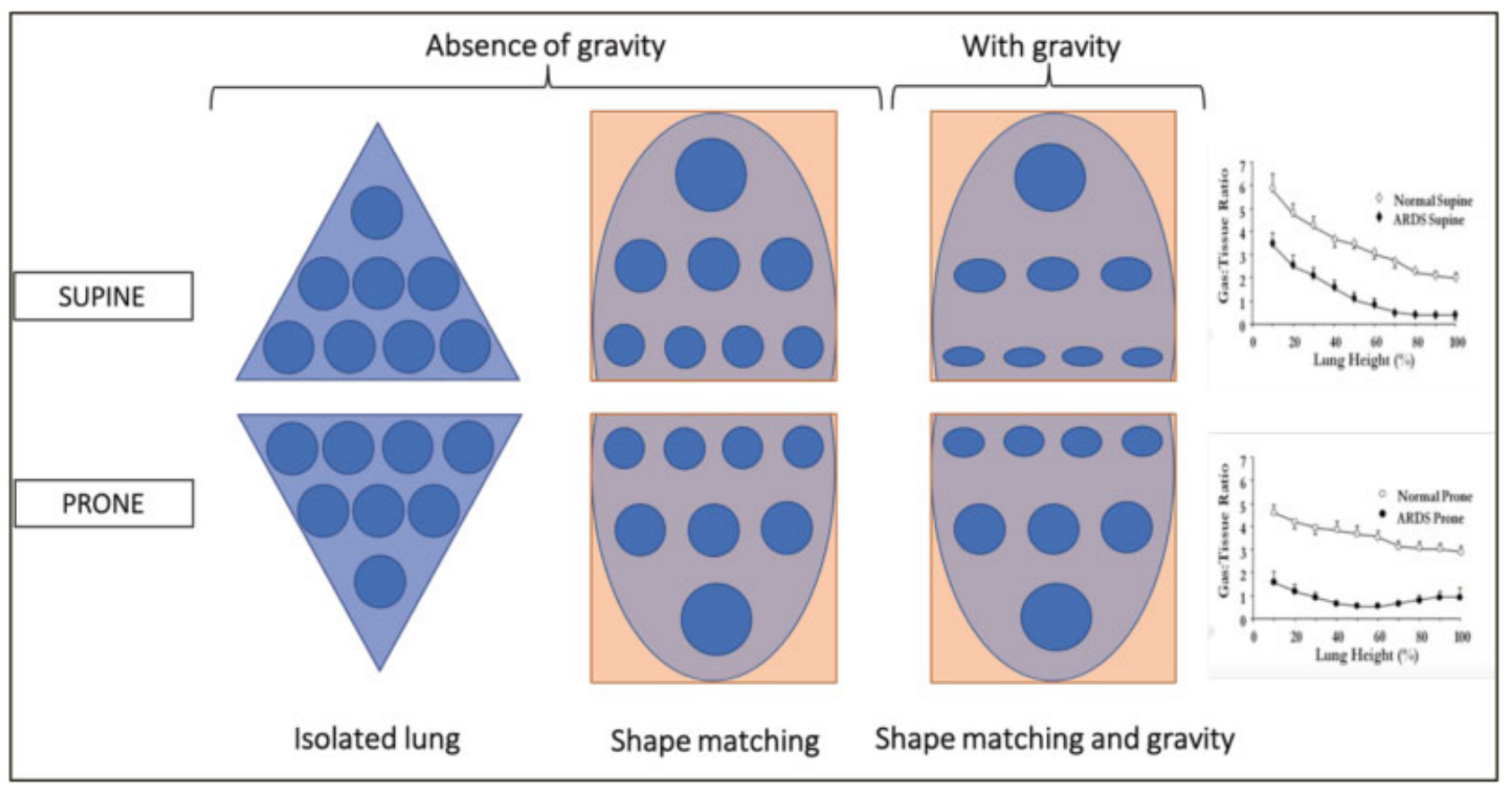

Fig. 2 Schematic representation of shape mismatch and gravitational effect. The isolated lung can be thought of as a cone, with alveolar units of the same size throughout the parenchyma if no gravity is hypothesized. When the lung is placed into the thoracic cage, the need of the cone to adapt to a more cylindrical shape imposes a stretch at the apex of the cone, which leads to an increase in size of the units in this area. When gravity is added, the units in the lower part of the lung feel the superimposed pressure of the units above and tend to collapse. If the patient is then pronated, the gravitational effect and the shape mismatch act in opposite directions, leading to a more homogeneous distribution of ventilation.

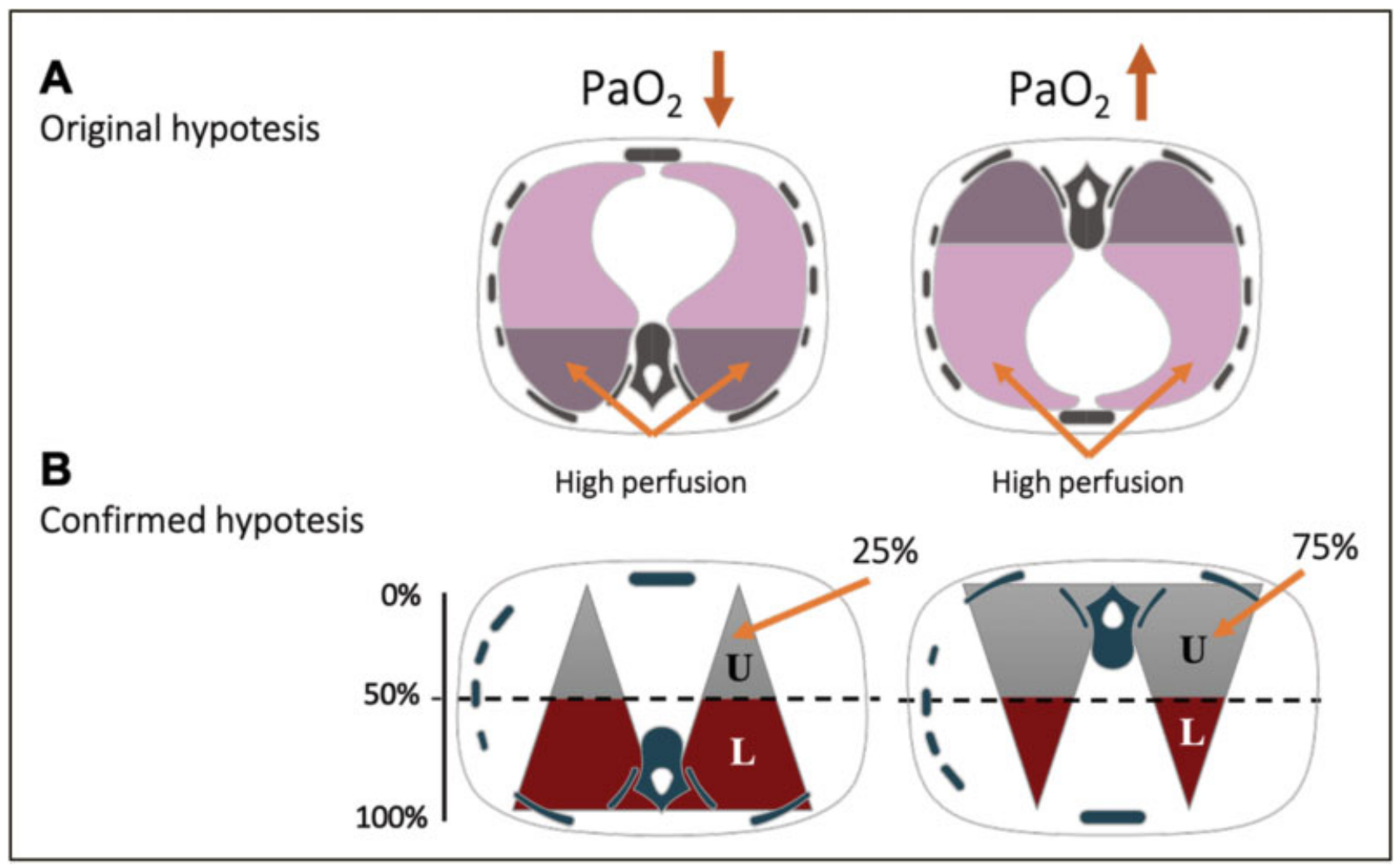

Fig. 3 The original explanation (panel A) for the improvement of oxygenation was that the ventral regions that were thought to be not affected by ARDS when the patient was pronated may receive more blood flow maximizing the $V / Q$ ratio. The confirmed hypothesis is instead the one showed in panel $B$. The amount of tissue in the dorsal regions of the lung is actually greater than the one in the ventral part. Hypothesizing a superimposed pressure capable of making the lung collapse below $50 \%$ of its height, it is clear that if the dorsal $50 \%$ becomes nondependent, the amount of tissue recruited is greater than the one derecruited in the ventral part (now dependent) (panel B: $\mathrm{U}$, upper; L, lower). 
Therefore, what actually accounts for the effect observed is the difference between the recruitment of the dorsal and the de-recruitment of the ventral parts of the lung. This difference is strictly related to the changes in shunt fraction as the perfusion, quite unexpectedly, has been found to be mostly unchanged between the prone and supine positions, as shown both experimentally ${ }^{14}$ and in humans. ${ }^{15-17}$ The role of the shape of the lung is quite obvious if we think about the sponge model (-Fig. 3). Imagine that the lung weight is increased to an amount such as to cause the compression of the dorsal lung regions at $50 \%$ of the lung height: due to the weight-related compressive forces when the patient is turned prone, the lung collapses at $50 \%$ of its height (the ventral region) while the regions above the $50 \%$ of the height (the dorsal regions) will reopen. The effect on gas exchange will depend on the amount of mass which is present in the upper $50 \%$ and the lower $50 \%$. If the lungs were perfectly round, the amount of mass would be exactly the same in the upper and lower $50 \%{ }^{18,19}$ However, since the shape is mostly conical (with the apex toward the ventral part of the thoracic cage), more lung mass is present in the dorsal $50 \%$, and this will reopen in the prone position. For example, a patient with pectus carinatum is the perfect responder to the prone position. Therefore, at least, part of the response associated with the prone position is strictly dependent on the anatomical morphology of the patient. Additionally, the role of the weight of the heart in dictating possible atelectasis also has to be taken into account ${ }^{20}$ : its compression is particularly relevant on the left lower lobe when the patient is in the supine position, whereas in the prone position, this effect disappears. $^{7}$

\section{Chest Wall Elastance}

In the supine position, the chest wall compliance is determined by the relative elasticity of the anterior chest wall and the diaphragm, since, the posterior portion of the thoracic cage is in contact with the bed. In the prone position, the overall diaphragm elasticity does not change, whereas the dorsal part of the thorax is free to move. Due to the anatomical conformation of the thorax, the dorsal chest wall is less compliant than the anterior one; therefore, the overall chest wall compliance decreases in a patient in the prone position. The consequence is a better distribution of gases toward the ventral and paradiaphragmatic lung regions, with a higher recruitment of these areas. It follows that the gas distribution becomes more homogeneous. ${ }^{21}$ Interestingly, a similar effect on gas exchange with a consequent increase in oxygenation may be observed by placing weights on the anterior portion of the thorax in the supine position (unpublished data). This maneuver decreases the distribution of the gases to the ventral regions while increasing it to the most dependent ones. The changes in the overall thoracic compliance during the prone position explain why this maneuver is associated with an increase in lung recruitability. The response of the respiratory system mechanics to the prone position would be an increase in airway pressure during volume-controlled ventilation or a decrease in tidal volume during pressure-controlled ventilation. Actually, after the maneuver, the plateau pressure may not increase but could remain constant or even decrease strongly: this would suggest a net lung recruitment during volume-controlled ventilation. Conversely, during pressure controlled ventilation, if the prone position is associated with a net lung recruitment, the tidal volume would remain constant or even increase.

\section{$\mathrm{CO}_{2}$ Clearance}

The $\mathrm{P}_{\mathrm{a}} \mathrm{CO}_{2}$ during the prone position may remain unchanged, increase, or even decrease. The behavior of $\mathrm{PaCO}_{2}$ depends on the behavior of the alveolar ventilation and its ratio to the total ventilated lung volume (i.e., with net lung recruitability). An increase in $\mathrm{P}_{2} \mathrm{CO}_{2}$ in the prone position may depend on the following two different mechanisms:

- Decreased alveolar ventilation (overinflated pulmonary units have a lower compliance and are therefore less ventilated; an uncorrected decrease of chest wall compliance may also account for a decrease in alveolar ventilation).

- A relative change on lung perfusion.

In contrast, while a decrease in $\mathrm{P}_{2} \mathrm{CO}_{2}$ has been found to be related to a net increase in recruitability, the increase in $\mathrm{P}_{a} \mathrm{O}_{2}$ is not totally dependent on this effect: oxygenation may indeed improve causes other than recruitability. ${ }^{22}$ In general, a decrease in $\mathrm{P}_{\mathrm{a}} \mathrm{CO}_{2}$ means a decrease in dead space. It is interesting to note that the physiological dead space, as computed by standard methods, includes not only the real dead space but also, in part, the shunt fraction component that increases the differences between the alveolar and arterial $\mathrm{PCO}_{2}$. Therefore, the dead space reflects the overall behavior of the respiratory gas exchange, and this may explain why this is one of the variables that is more associated with the final outcome. This has clearly been shown in ARDS. ${ }^{23}$ We additionally found that a decrease in $\mathrm{P}_{\mathrm{a}} \mathrm{CO}_{2}$ in response to prone positioning is associated with a reduction in 28-day mortality. ${ }^{24,25}$

\section{Clinical Trials}

It is interesting to show how the clinical trials have followed the evolution of the pathophysiological knowledge concerning ARDS. Actually, randomized trials are large-scale deductive experiments whose development might be summarized in the following steps ${ }^{26}$ :

1. A background theory is introduced from the results of several observations.

2. The theory generates a hypothesis.

3. The hypothesis is experimentally tested in a clinical trial, which should be properly designed and feasible.

4. If the experiment is "negative," that is, the hypothesis is not proved, then the theory has to be revised.

Subsequently, we will examine the sequence of trials on the prone position, analyzing them according to the theories that were believed to be true or were to be proven and discussing the feasibility of their experimental designs. 


\section{Gattinoni et al $^{27}$}

This study, published in 2001, was actually designed in 1995 and performed between 1996 and 1999. The theories behind the trial were straightforward: better oxygenation is associated with better survival, and prone positioning improves oxygenation. The hypothesis was that prone positioning would improve survival. The design reflected the knowledge of the time: the protocol imposed 6 hours of the prone position per day, a period of time primarily selected according to nurse shifts. The sample size was designed to detect a $20 \%$ decrease in mortality in the prone position group. The study was stopped because of the slow rate of patient recruitment. It is worth noting that at the time of the study, the common ventilator setup implied $10 \mathrm{~mL} / \mathrm{kg}$ of tidal volume: the so-called protective ventilation had still to be proposed. This study, however, clearly showed that the background theory (better oxygenation = better survival) was wrong. Prone position was indeed associated with a better oxygenation but with no benefits on survival. It is interesting to note that even if the trial was overall negative, some advantages of the prone position could be observed in the quartile with the lowest $\mathrm{P}_{\mathrm{a}} \mathrm{O}_{2} / \mathrm{FiO}_{2}$ ratio.

\section{Guérin et $\mathbf{a l}^{28}$}

This trial was designed approximately at the same time of the previous one, and the knowledge of ARDS pathophysiology was the same. Indeed, the theories behind the trial and the hypothesis were the same as in the study of Gattinoni et al. The results disproved the hypothesis and the theory behind it. Again, the prone position improved oxygenation, but mortality remained the same in the treatment and control arms. Interestingly, not only ARDS patients but also patients with hypoxemic acute respiratory failure were treated. Therefore, strictly speaking, the first two trials did not show that the prone position "does not work," but they showed that a better oxygenation per se, with the other conditions being similar and within the time frame used in the two trials, does not lead to an improvement in outcome. Note that the ARMA (Acute Respiratory Management in ARDS) trial performed some years later ${ }^{29}$ and based on different theoretical background clearly demonstrated that improvement of oxygenation is not associated with an improved survival.

\section{Mancebo et $\mathbf{a l}^{30}$}

The design of the trial from the Spanish group represents a breakthrough in the theories behind the trials on the prone position. Indeed, although the ARMA trial results were still not available at the time when the study was designed, there was already a progressive awareness on the potentially damaging effects of high volume and pressure during mechanical ventilation. Therefore, since the oxygenation but not the mechanical ventilation per se was responsible for a fraction of the mortality in ARDS patients, it seemed logical to prolong the possible "protection" provided by prone positioning for as long as possible. As a consequence, the prone position was applied for at least 20 hours; the plateau pressure was kept below $35 \mathrm{~cm} \mathrm{H}_{2} \mathrm{O}$ or to $40 \mathrm{~cm} \mathrm{H}_{2} \mathrm{O}$ if chest wall stiffness was suspected and/or tidal volume below $10 \mathrm{~mL} / \mathrm{kg}$. This study was interrupted for enrolment difficulties, but the message was quite strong and in favor of the prone position (mortality $58 \%$ in the supine group vs. $43 \%$ in the prone group; $p=0.12$ ).

\section{Taccone et al ${ }^{31}$}

In this study, we tried to apply the best of the knowledge available: the theory behind this study was definitely based on lung mechanics. Indeed, when the trial was designed, it was quite clear that the possible advantages of prone positioning were because of an increase in lung homogeneity, with a more even distribution of stress and strain. ${ }^{32-34}$ Knowing the difficulties associated with the enrollment

Table 1 Summary of the five major trials on the prone position

\begin{tabular}{|l|l|l|l|l|l|}
\hline Clinical Trials in the Prone Positioning \\
\hline Year & $\mathbf{2 0 0 1}$ & $\mathbf{2 0 0 4}$ & $\mathbf{2 0 0 6}$ & $\mathbf{2 0 0 9}$ & 2013 \\
\hline & Gattinoni et al. & Guérin et al. & Mancebo et al. & Taccone et al. & Guérin et al. \\
\hline Study period & $1996-1999$ & $1998-2002$ & $1998-2002$ & $2004-2008$ & $2008-2011$ \\
\hline Patients & 304 & 802 & 142 & 344 & 466 \\
\hline Average $\mathrm{PaO}_{2} / \mathrm{FiO}_{2}$ at enrollment & 127 & 152 & 105 & 113 & 100 \\
\hline PEEP at enrollment & 10 & 8 & 7 & 10 & 10 \\
\hline SAPS II & 40 & 46 & 41 & 41 & 46 \\
\hline Duration of prone position & $7 \mathrm{~h} \times 5 \mathrm{~d}$ & $9 \mathrm{~h} \times 4 \mathrm{~d}$ & $17 \mathrm{~h} \times 10 \mathrm{~d}$ & $18 \mathrm{~h} \times 8 \mathrm{~d}$ & $17 \mathrm{~h} \times 4 \mathrm{~d}$ \\
\hline Protective ventilation & $\mathrm{No}$ & $\mathrm{No}$ & $\mathrm{VT}<10 \mathrm{~mL} / \mathrm{kg}$ & $\mathrm{VT}<10 \mathrm{~mL} / \mathrm{kg}$ & $6 \mathrm{~mL} / \mathrm{kg}$ \\
\hline Follow-up & $6 \mathrm{mo}$ & $90 \mathrm{~d}$ & Hospital discharge & $6 \mathrm{mo}$ & $90 \mathrm{~d}$ \\
\hline Mortality (\%) & & & & & \\
\hline Supine & 58.3 & 42.2 & 60 & 52.9 & 41 \\
\hline Prone & 62.2 & 43.3 & 50 & 47.6 & 23.6 \\
\hline$p$-Value & 0.5 & 0.74 & 0.22 & 0.33 & 0.001 \\
\hline
\end{tabular}


(which in all trials amount approximately to 0.25 patients per month per unit), we decided to use the same protocol as in the Spanish trial and planned in advance to eventually merge our study with Mancebo et al's study if the number of patients had been too small. Actually, this study per se showed a nonsignificant reduction in mortality with the prone position, but merging with Mancebo et al's data demonstrated that the prone position provided a survival advantage in most severe patients. ${ }^{35}$ However, the reviewers of the major journals refused this approach, and this study was published as "a negative trial." What seemed clear, however, was that the signal in favor of the prone position was strong in severe ARDS patients, whereas in the moderate ARDS patients, no advantage was detected.

\section{Guérin et $\mathbf{a l}^{36}$}

In this study, the investigators applied all the best conditions to detect a significant advantage associated with the prone position. The patients selected had $\mathrm{P}_{\mathrm{a}} \mathrm{O}_{2} / \mathrm{FiO}_{2}$ below $150 \mathrm{~mm}$ $\mathrm{Hg}$; therefore, the moderate ARDS category was split into two categories $\left(\mathrm{P}_{\mathrm{a}} \mathrm{O}_{2} / \mathrm{FiO}_{2}\right.$ values below $150 \mathrm{~mm} \mathrm{Hg}$ are associated with well-defined pathological and clinical findings, ${ }^{37}$ whose severity differs from that of ARDS patients classified as moderate according to the Berlin definition ${ }^{38}$ ). The intensive care units who took part in the trial had a documented experience in proning, and the protective lung ventilation was strictly applied. The results documented a clear benefit of proning compared to the supine position in this accurately selected population. The characteristics of all these trials are summarized in - Table 1.

\section{Indications}

We believe that the indications for the prone position is straightforward: the condition of the patients must be so severe that a standard mechanical ventilation in the supine position may lead, in a significant amount of them, to a significant increase in mortality due to regional lung stress and strain.

It is worth noting that according to the actual knowledge, mechanical ventilation could also be part of the therapy of patients with extracorporeal support. Whatever the pattern of ventilation might be, the increase in lung homogeneity following the prone position will decrease its potential harms.

\section{Contraindications}

The prone position as a long-term therapy is useless in patients in which the anatomical and severity characteristics allow a standard mechanical ventilation $\left(\mathrm{P}_{\mathrm{a}} \mathrm{O}_{2} / \mathrm{FiO}_{2}>150\right.$ $\mathrm{mm} \mathrm{Hg}$ ). Under these conditions, the possible risks associated with prone positioning (accidental extubation, pressure ulcers, catheter displacement, need for increased sedation $^{39}$ ) overcome its advantages that are only evident when the "baby lung" is so small that even a "protective" ventilation is harmful in the supine position.

Conflict of Interest

None.

\section{References}

1 Piehl MA, Brown RS. Use of extreme position changes in acute respiratory failure. Crit Care Med 1976;4(01):13-14

2 Douglas WW, Rehder K, Beynen FM, Sessler AD, Marsh HM. Improved oxygenation in patients with acute respiratory failure: the prone position. Am Rev Respir Dis 1977;115(04): 559-566

3 Maunder RJ, Shuman WP, McHugh JW, Marglin SI, Butler J. Preservation of normal lung regions in the adult respiratory distress syndrome. Analysis by computed tomography. JAMA 1986;255(18):2463-2465

4 Gattinoni L, Mascheroni D, Torresin A, et al. Morphological response to positive end expiratory pressure in acute respiratory failure. Computerized tomography study. Intensive Care Med 1986;12(03):137-142

5 Gattinoni L, Pesenti A, Avalli L, Rossi F, Bombino M. Pressurevolume curve of total respiratory system in acute respiratory failure. Computed tomographic scan study. Am Rev Respir Dis 1987;136(03):730-736

6 Langer M, Mascheroni D, Marcolin R, Gattinoni L. The prone position in ARDS patients. A clinical study. Chest 1988;94(01): 103-107

7 Wiener CM, McKenna WJ, Myers MJ, Lavender JP, Hughes JM. Left lower lobe ventilation is reduced in patients with cardiomegaly in the supine but not the prone position. Am Rev Respir Dis 1990; 141(01):150-155

8 Richter T, Bellani G, Scott Harris R, et al. Effect of prone position on regional shunt, aeration, and perfusion in experimental acute lung injury. Am J Respir Crit Care Med 2005;172(04):480-487

9 Bone RC. The ARDS lung. New insights from computed tomography. JAMA 1993;269(16):2134-2135

10 Gattinoni L, D'Andrea L, Pelosi P, Vitale G, Pesenti A, Fumagalli R. Regional effects and mechanism of positive end-expiratory pressure in early adult respiratory distress syndrome. JAMA 1993;269 (16):2122-2127

11 Pelosi P, D’Andrea L, Vitale G, Pesenti A, Gattinoni L. Vertical gradient of regional lung inflation in adult respiratory distress syndrome. Am J Respir Crit Care Med 1994;149(01):8-13

12 Hubmayr RD. Perspective on lung injury and recruitment: a skeptical look at the opening and collapse story. Am J Respir Crit Care Med 2002;165(12):1647-1653

13 Gattinoni L, Taccone P, Carlesso E, Marini JJ. Prone position in acute respiratory distress syndrome. Rationale, indications, and limits. Am J Respir Crit Care Med 2013;188(11):1286-1293

14 Wiener CM, Kirk W, Albert RK. Prone position reverses gravitational distribution of perfusion in dog lungs with oleic acidinduced injury. J Appl Physiol (1985) 1990;68(04):1386-1392

15 Lamm WJ, Graham MM, Albert RK. Mechanism by which the prone position improves oxygenation in acute lung injury. Am J Respir Crit Care Med 1994;150(01):184-193

16 Nyrén S, Mure M, Jacobsson H, Larsson SA, Lindahl SG. Pulmonary perfusion is more uniform in the prone than in the supine position: scintigraphy in healthy humans. J Appl Physiol (1985) 1999;86(04):1135-1141

17 Henderson AC, Sá RC, Theilmann RJ, Buxton RB, Prisk GK, Hopkins SR. The gravitational distribution of ventilation-perfusion ratio is more uniform in prone than supine posture in the normal human lung. J Appl Physiol (1985) 2013;115(03): 313-324

18 Tobin MJ. Principles and Practice of Mechanical Ventilation. 3rd ed. New York, NY: McGraw-Hill Medical; 2013

19 Cornejo RA, Díaz JC, Tobar EA, et al. Effects of prone positioning on lung protection in patients with acute respiratory distress syndrome. Am J Respir Crit Care Med 2013;188(04):440-448

20 Albert RK, Hubmayr RD. The prone position eliminates compression of the lungs by the heart. Am J Respir Crit Care Med 2000;161 (05):1660-1665 
21 Pelosi P, Tubiolo D, Mascheroni D, et al. Effects of the prone position on respiratory mechanics and gas exchange during acute lung injury. Am J Respir Crit Care Med 1998;157(02):387-393

22 Protti A, Chiumello D, Cressoni M, et al. Relationship between gas exchange response to prone position and lung recruitability during acute respiratory failure. Intensive Care Med 2009;35 (06):1011-1017

23 Nuckton TJ, Alonso JA, Kallet RH, et al. Pulmonary dead-space fraction as a risk factor for death in the acute respiratory distress syndrome. N Engl J Med 2002;346(17):1281-1286

24 Gattinoni L, Vagginelli F, Carlesso E, et al; Prone-Supine Study Group. Decrease in $\mathrm{PaCO} 2$ with prone position is predictive of improved outcome in acute respiratory distress syndrome. Crit Care Med 2003;31(12):2727-2733

25 Raurich JM, Vilar M, Colomar A, et al. Prognostic value of the pulmonary dead-space fraction during the early and intermediate phases of acute respiratory distress syndrome. Respir Care 2010; 55(03):282-287

26 Gattinoni L, Giomarelli P. Acquiring knowledge in intensive care: merits and pitfalls of randomized controlled trials. Intensive Care Med 2015;41(08):1460-1464

27 Gattinoni L, Tognoni G, Pesenti A, et al; Prone-Supine Study Group. Effect of prone positioning on the survival of patients with acute respiratory failure. N Engl J Med 2001;345(08): 568-573

28 Guérin C, Gaillard S, Lemasson S, et al. Effects of systematic prone positioning in hypoxemic acute respiratory failure: a randomized controlled trial. JAMA 2004;292(19):2379-2387

29 Brower RG, Matthay MA, Morris A, Schoenfeld D, Thompson BT, Wheeler A; Acute Respiratory Distress Syndrome Network. Ventilation with lower tidal volumes as compared with traditional tidal volumes for acute lung injury and the acute respiratory distress syndrome. N Engl J Med 2000;342(18):1301-1308
30 Mancebo J, Fernández R, Blanch L, et al. A multicenter trial of prolonged prone ventilation in severe acute respiratory distress syndrome. Am J Respir Crit Care Med 2006;173(11):1233-1239

31 Taccone P, Pesenti A, Latini R, et al; Prone-Supine II Study Group. Prone positioning in patients with moderate and severe acute respiratory distress syndrome: a randomized controlled trial. JAMA 2009;302(18):1977-1984

32 Chiumello D, Carlesso E, Cadringher P, et al. Lung stress and strain during mechanical ventilation for acute respiratory distress syndrome. Am J Respir Crit Care Med 2008;178(04):346-355

33 Perchiazzi G, Rylander C, Vena A, et al. Lung regional stress and strain as a function of posture and ventilatory mode. J Appl Physiol (1985) 2011;110(05):1374-1383

34 Valenza F, Guglielmi M, Maffioletti M, et al. Prone position delays the progression of ventilator-induced lung injury in rats: does lung strain distribution play a role? Crit Care Med 2005;33(02): 361-367

35 Gattinoni L, Carlesso E, Taccone P, Polli F, Guérin C, Mancebo J. Prone positioning improves survival in severe ARDS: a pathophysiologic review and individual patient meta-analysis. Minerva Anestesiol 2010;76(06):448-454

36 Guérin C, Reignier J, Richard JC, et al; PROSEVA Study Group. Prone positioning in severe acute respiratory distress syndrome. $\mathrm{N}$ Engl J Med 2013;368(23):2159-2168

37 Maiolo G, Collino F, Vasques F, et al. Reclassifying acute respiratory distress syndrome. Am J Respir Crit Care Med 2018;197(12): 1586-1595

38 Ranieri VM, Rubenfeld GD, Thompson BT, et al; ARDS Definition Task Force. Acute respiratory distress syndrome: the Berlin Definition. JAMA 2012;307(23):2526-2533

39 Messerole E, Peine P, Wittkopp S, Marini JJ, Albert RK. The pragmatics of prone positioning. Am J Respir Crit Care Med 2002;165(10):1359-1363 